\title{
Tourisme et crises sanitaires mondiales dans l'Histoire : véritablement un impensé ?
}

Tourism and Global Health Crises in History: Is It Truly an Unthought?

Yves-Marie Evanno et Johan Vincent

\section{OpenEdition}

Journals

Édition électronique

URL : https://journals.openedition.org/tourisme/3795

DOI : 10.4000/tourisme.3795

ISSN : 2492-7503

Éditeur

Association Mondes du tourisme

Référence électronique

Yves-Marie Evanno et Johan Vincent, « Tourisme et crises sanitaires mondiales dans l'Histoire: véritablement un impensé ? », Mondes du Tourisme [En ligne], 20 | 2021, mis en ligne le 15 décembre 2021, consulté le 17 décembre 2021. URL : http://journals.openedition.org/tourisme/3795; DOI : https://doi.org/10.4000/tourisme.3795

Ce document a été généré automatiquement le 17 décembre 2021.

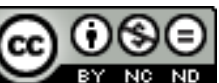

Mondes du tourisme est mis à disposition selon les termes de la licence Creative Commons Attribution - Pas d'Utilisation Commerciale - Pas de Modification 4.0 International. 


\section{Tourisme et crises sanitaires mondiales dans l'Histoire : véritablement un impensé ?}

Tourism and Global Health Crises in History: Is It Truly an Unthought?

Yves-Marie Evanno et Johan Vincent

1 Comble de l'activité d'agrément, faire du tourisme n'est pas une pratique dépourvue de risques. En effet, aléas naturels, conflits militaires, tensions sociales, ou encore risques sanitaires troublent en permanence la carte du tourisme international (Dehoorne, 2013). À ce titre, la crise de la Covid-19 n'a fait que rappeler à quel point les épidémies peuvent fragiliser le secteur touristique. Or, si l'évènement est particulièrement marquant par son ampleur, il n'est en réalité pas unique à l'échelle de l'histoire. Depuis près de deux siècles, le monde a connu de nombreuses épidémies qui, au moins à l'échelle locale, ont impacté plus ou moins durablement les territoires (Trainar, 2020).

2 Pourtant, leurs conséquences sur le tourisme demeurent largement méconnues. Jusqu'à récemment, les études scientifiques historiques s'intéressant à l'impact des crises sanitaires sur le tourisme sont restées peu nombreuses, comme le déploraient notamment Marina Novelli et ses coauteurs en 2018. Quand elles existent, ces études mélangent les crises sanitaires avec d'autres crises liées à l'économie, au terrorisme, aux catastrophes naturelles (Hall, 2010 ; Teitler-Regev et al., 2013), ou abordent des sujets connexes, en particulier le rôle des médias (Baxter et Bowen, 2004 ; Monterrubio, 2010). En outre, elles sont généralement axées sur la dimension du tourisme international, qui sert de référence à toute réflexion. La crise de la Covid-19, surtout depuis la mise en place des confinements à l'échelle nationale à partir de mars 2020, a soudainement changé la donne: elle a été lourdement investie par le milieu de la recherche, et ce toutes disciplines scientifiques confondues. Dès lors, les numéros spéciaux des revues en tourisme se sont multipliés - parfois dans l'urgence - afin d'évoquer une crise qualifiée de "sans précédent ", impression renforcée à la lumière d'autres pandémies récentes (SRAS, grippe aviaire, grippe porcine, MERS...) dont les effets auraient été peu durables sur le tourisme (Karabulut et al., 2020). 
3 Pourtant, une approche historique sur le temps long, au-delà des seuls épisodes du $\mathrm{XXI}^{\mathrm{e}}$ siècle souvent évoqués, permet de tempérer une telle conclusion. Il n'existe pas de « crises » sans effets et, systématiquement, les acteurs du tourisme doivent s'adapter face à ces épisodes exceptionnels. Les crises sanitaires étudiées dans le cadre de cet article engloberont aussi bien les épidémies que les pandémies, tant la nuance entre les deux reste difficile à saisir, la principale différence résidant dans l'ampleur géographique d'une maladie infectieuse. Une épidémie (du latin epidemia dérivé du grec ancien qui signifie "à la maison ») se limiterait à une région, à un pays ou à une zone bien définie alors qu'une pandémie (du grec ancien pan, qui signifie «tout», et demos, qui signifie " peuple ») est une épidémie à plusieurs foyers, s'étendant à la population d'un continent, voire au monde entier. En conséquence, limiter notre propos à l'une ou à l'autre nous semblait inopérant dans la mesure où seule la "crise sanitaire ", qui en découle, a de potentiels effets sur le tourisme. Notre propos s'intéressera alors aux comportements des acteurs du secteur touristique face aux crises sanitaires afin de déceler des permanences dans la gestion de ces dernières et ainsi, par contrecoup, de mieux comprendre les mutations.

Pour comprendre ces enjeux, il est nécessaire de travailler la question en remontant le temps jusqu'au premier quart du $\mathrm{xIX}^{\mathrm{e}}$ siècle, quand les vieilles "pestes " semblaient avoir regagné d'elles-mêmes leurs lointaines niches, et d'étudier le sujet jusqu'au début des années 2020. Ce travail se veut être, d'abord, une première synthèse menée à partir de la littérature existante et de quelques dépouillements d'archives réalisés essentiellement à partir de la presse européenne et des archives françaises de surveillance des populations. Parce qu'il souhaite avant tout nourrir la réflexion sur le lien entre crises sanitaires et tourisme, cet article lancera quelques pistes prospectives dont les historiens pourront se saisir afin de renouveler profondément nos connaissances sur le sujet. Pour ce faire, il faudra mobiliser des sources inédites et parfois, difficiles à trouver puisque les classements archivistiques ne sont pas constitués par thématiques mais par service versant. Cela explique, au moins en partie, pourquoi le sujet n'a jamais réellement été abordé sur le long terme. Enfin, notre propos, qui se veut mondial, sera en conséquence majoritairement axé sur des exemples européens.

\section{La fin de l'immunité universelle sanitaire des stations de tourisme au XIX ${ }^{\mathrm{e}}$ siècle}

5 Il est de coutume de considérer le xix ${ }^{\mathrm{e}}$ siècle comme la période fondamentale durant laquelle se développent et se diversifient les pratiques touristiques. Or, cet essor n'est pas linéaire, le secteur du tourisme étant notamment marqué par de nombreuses crises, y compris sanitaires. Les pandémies - en particulier le choléra - ont joué un rôle essentiel dans ces évolutions en modifiant les flux et les intentions touristiques, tout en favorisant la prise de conscience que la respectabilité des territoires est devenue indispensable.

\section{La gestion des pandémies au XIX siècle : une entrave aux voyages ?}

$\mathrm{Au} \mathrm{XIX}^{\mathrm{e}}$ siècle, alors que les pratiques touristiques se développent dans le monde, un nouvel ennemi surgit : le choléra. Responsable d'épisodes épidémiques et pandémiques 
réguliers, tout au long du siècle, le choléra encourage les mesures policières fortes pour contenir la diffusion d'un danger systématiquement présenté comme venant de l'extérieur. Le voyageur, sans distinction de ses motivations (agrément ou commerce), est perçu comme une menace potentielle. Le préjugé n'est en réalité pas nouveau. Depuis le Moyen Âge, et en particulier après la Peste noire (xIV siècle), des quarantaines sont mises en place pour les voyageurs arrivés par la mer (Houdaille, 1975).

7 Dans la continuité des siècles précédents, la gestion de la crise sanitaire reste donc principalement policière. Le "cordon sanitaire » devient l'un des outils privilégiés de réponse à la crise. Apparu dans le langage de l'action publique en 1821 au moment d'une épidémie de fièvre jaune sur la frontière franco-espagnole, il est mis en place à de multiples reprises. Ainsi, en 1831, durant la Deuxième pandémie de choléra (1827-1837), le gouvernement de Sardaigne crée un Giunta Superiore di Sanità pubblica au moment où, en France, Casimir Perier réactive les dispositions de la loi de 1822 sur les contrôles sanitaires aux frontières (Tabureau, 2007). Ces mesures perturbent les projets des voyageurs, comme le souligne Stendhal dans les Mémoires d'un touriste. Selon l'auteur français, le voyageur ne doit pas prendre au tragique les accidents de quarantaine qui, entre autres choses, viennent "souvent contrarier les plus jolies courses" (Stendhal, 1929 [1838], p. 457). De la même manière, le cordon sanitaire mis en place en 1834 par le comté de Nice le long de la rivière du Var, en contrariant les plans d'Henry Brougham, joue un rôle insoupçonné dans le développement du tourisme local. En effet, c'est à cette occasion qu'il découvre le petit village de pêcheurs de Cannes dont il fait, par la suite, un haut lieu de villégiature.

8 À Nice, comme ailleurs, ce dispositif de quarantaine porte préjudice au commerce marchand (Bon, 2018 ; Bachemont, 2011) et, par la force des choses, au tourisme. Dès lors, cette gestion de la crise sanitaire est contestée. En 1835, le Consul de France proteste ainsi contre la fermeture de la frontière car le danger ne vient pas, selon lui, du Var, mais des émigrations de Gênes. Quasiment cinquante ans plus tard (lors de la crise sanitaire parfois décrite seulement comme une épidémie, parfois comme un épisode de la Cinquième pandémie de choléra), la presse française, comme La République française du 22 septembre 1884, dénonce encore « les mesures tyranniques et absurdes prises aux gares frontières par les agents galonnés » (Hanus, 2020, p. 8) contre ceux qui franchissent la frontière franco-italienne près de Menton. Sous le regard des journalistes de L'Illustration (Anonyme, 1884), ils sont présentés comme subissant les mesures de confinement préventif et de décontamination dans les baraquements du Lazaret de la Latta.

Du fait de la variabilité des mesures d'un pays à l'autre, les déplacements en Europe ne sont pas facilités au moment de ces épidémies et pandémies, alors que l'importance de voyager à certaines occasions est reconnue. Lors de l'Exposition universelle de 1851, propice à un grand nombre de déplacements dans toute l'Europe tandis que sévit une nouvelle pandémie de choléra (la troisième), une conférence réunissant douze grandes puissances tente bien d'uniformiser les règlements de protection sanitaire, mais seuls trois pays (France, Sardaigne et Portugal, bien que ce dernier n'ait, dans les faits, jamais ratifié ladite "Convention sanitaire internationale ») parviennent à s'entendre au sujet de trois maladies: choléra, peste et fièvre jaune (Howard-Jones, 1975, p.13-18; Houdaille, 1975). 


\section{Les stations balnéaires comme zones refuges?}

10 Avant la découverte du microbe (dénomination proposée par CharlesEmmanuel Sédillot en 1878) et la révolution microbienne qui y est associée (Louis Pasteur et les scientifiques de l'époque prouvant que les microbes sont partout), la maladie est perçue comme la conséquence d'un mauvais environnement. Changer d'environnement, c'est donc espérer pouvoir échapper à la maladie, et bien sûr aux épidémies et aux pandémies. Ainsi, lorsque le choléra touche Paris en avril 1832, les parlementaires se pressent de quitter la capitale pour leur département. Au même moment, dans le Var, il faut suspendre les congés des fonctionnaires en débandade. L'année suivante, à Aix et dans plusieurs villes du Midi, les hôtels urbains sont abandonnés - symboliquement - aux domestiques afin que leurs propriétaires puissent gagner leurs maisons des champs (Delumeau et Lequin, 2020). Au même moment, des hivernants prolongent leur séjour dans les stations balnéaires méditerranéennes (Archives de Nice, 2020) alors que le climat y est habituellement jugé délétère durant les mois d'été. La récurrence de ces fuites influence le développement du tourisme, comme le souligne Helen Davies (2015) qui fait, par exemple, le rapprochement entre l'épisode cholérique parisien de 1832 et le début des tractations pour la création de la ligne Paris-Saint-Germain-en-Laye, première ligne de voyageurs ouverte en France sur des arguments touristiques.

11 Le bord de mer s'impose comme l'un des refuges privilégiés des élites, offrant la possibilité de bains dans les vagues, alors que la montagne est considérée comme un espace à garder à distance - l'essor spécifique du thermalisme, renouvelé par le vitalisme à partir de 1778 (Nouvel, 2011), profite de l'engouement pour les spectacles de nature qui touchent l'âme - jusqu'à l'essor du climatisme, dans les années 1840 (GranetAbisset, 2011). Jusqu'au milieu du $\mathrm{XIX}^{\mathrm{e}}$ siècle persiste une croyance qui veut que les populations des côtes seraient immunisées contre les maladies de "civilisation", considérées comme communes en ville et qu'un séjour sur le littoral éloignerait naturellement. En 1817, Jane Austen, dans son roman inachevé Sanditon, évoque cette croyance à travers son héros, Mr. Parker, propriétaire de divers magasins et de pensions dans une station balnéaire imaginaire, qui :

tenait pour certain que personne ne pouvait vraiment bien se porter, que personne (même si les secours fortuits de l'exercice et de la volonté peuvent momentanément donner à quelqu'un l'apparence de la santé) ne pouvait jouir d'une bonne santé réelle et permanente, sans passer au moins six semaines au bord de la mer chaque année. (cité par Porter, 1995, p. 37-38)

12 Ce mythe semble progressivement se fissurer au cours du XIX siècle avec la succession des pandémies de choléra, plus particulièrement durant la Troisième pandémie de choléra. En effet, la médiatisation de nombreuses contaminations dans plusieurs stations balnéaires porte un coup fatal à cette croyance immunitaire dans l'opinion, aussi bien au Royaume-Uni (dans les stations du Devon, selon Travis, 1993) qu'en France. Un épisode particulièrement symbolique intervient aux Sables-d'olonne (Vendée). Le 12 août 1849, au plus fort de la saison estivale, le choléra frappe ainsi une «foule de baigneurs » avant de se répandre à l'ensemble de la station, poussant la plupart des familles à quitter leur lieu de villégiature, qui ne joue plus son rôle immunisant (Anonyme, 1849, p. 2).

La crise de 1849 n'est pas seule responsable de ce changement de paradigme dans le monde occidental. Mieux nourris, à partir de la seconde moitié du XIX siècle, les 
Occidentaux sont également de mieux en mieux soignés. Si le discours médical continue de répéter l'argument donnant au changement d'air des vertus thérapeutiques, les prescriptions sont désormais individualisées : le choix du lieu doit être effectué suivant les indications médicales. Cette rupture rejoint la « découverte » des vertus de l'air sec et l'essor du climatisme, notamment encouragé par les travaux du docteur Alexander Taylor sur le climat palois, qui connaissent une grande notoriété après 1842 . Le discours médical tend à réduire les espaces de l'hivernage à quelques destinations à la fin du siècle, en France comme en Italie ; après 1863, le docteur Bennet, « inventeur " de Menton, prospecte le Sud de l'Europe en quête des winter climates (Boyer, 2002). En conséquence, la fonction thérapeutique s'amoindrit à partir du milieu du XIX siècle au profit de la fonction préventive associée, de plus en plus, à une pratique mondaine et à des activités ludiques (Toulier, 1996).

Le discours médical est recyclé pour afficher une moralisation des pratiques touristiques. La conquête des bords de mer et des espaces montagnards procède alors du désir d'associer le souci de la santé et celui des plaisirs mondains ou sportifs (Bertho-Lavenir, 1999). Un reliquat de discours sanitaire continue toujours d'habiller les justifications de déplacement. C'est ainsi qu'un guide de voyage local consacré à Petrópolis (Brésil) continue de vanter, en 1910, les mérites de la "Cité impériale " comme une "ville de guérison " (Empreza, 1910). De la même manière, en Europe, les rapports du service de santé des Armées rappellent encore en 1916 à quel point la «brise bienfaisante de mer» contribue au "rapide rétablissement des forces exténuées » des soldats blessés au front militaire (Evanno et Vincent, 2017, p. 187-188). Mais ce qui change, c'est que l'argument n'est plus universel et systématique. Le bouleversement thérapeutique du dernier quart du XIX siècle vient répondre aux exigences d'une population désormais médicalisée, moins par un encadrement compétent que par sa conversion à la cause de la santé (Delumeau et Lequin, 2020). La dimension locale est d'autant plus renforcée que l'espace géographique des maladies infectieuses ne cesse de se rétracter sur des foyers locaux ou régionaux, parfois dans certaines catégories de la population. Le monde du risque épidémique se confond progressivement avec celui d'une certaine marginalité (op. cit.).

\section{La préservation souhaitée de la respectabilité d'un territoire}

Le XIX ${ }^{e}$ siècle met - déjà - parfaitement en évidence le fait que les crises épidémiques et pandémiques, lorsqu'elles incriminent des territoires touristiques parfaitement identifiés, portent atteinte à la respectabilité de ces derniers. En effet, la réputation touristique d'un territoire est un capital fragile, et cela est d'autant plus vrai dans un contexte de crise qui favorise l'émergence et la diffusion des rumeurs (Flem, 1982). En 1839, c'est un risque d'épidémie de fièvre typhoïde qui fait peur à Biarritz, rendant désert le village ; en 1856, c'est cette fois la menace du choléra qui justifie la brièveté du séjour du couple impérial dans la station basque (Laborde, 2001). Pour les populations locales, il s'agit d'abord de prendre conscience de l'apparition de la maladie avant de tenter de la maîtriser. Au moment de la Première pandémie de choléra, l'attitude des autorités ne rassure pas ceux qui ne peuvent déroger au travail. Anxieux de ne pas ajouter au désordre et aux inquiétudes, souvent débordés par l'ampleur des prophylaxies à organiser, préfets et maires en arrivent à nier l'épidémie pour « calmer les imaginations » et dissiper « les bruits exagérés » (Delumeau et Lequin, 2020, p. 379). 
16 La prise de conscience de la nécessité de contrôler son action et sa communication pour ne pas mettre en péril l'imagerie touristique d'un lieu est donc ancienne. Déjà en 1849, dans le Devon, les autorités tentent de préserver l'image de la région pour ne pas pénaliser l'activité touristique (Travis, 1993). Alors que le choléra atteint Torquay en août, les journaux restent discrets sur l'épidémie. La presse locale soupçonne au départ une conspiration du silence. Il faut attendre le 5 septembre pour que le Torquay Directory annonce la mort de trois personnes. Les autres journaux réfutent pourtant cette annonce jusqu'à la fin du mois de septembre quand la situation épidémique devient incontrôlable. Le ministre de la Santé est averti qu'avec la panique née de la diffusion de la nouvelle, la partie de la ville où les morts sont les plus nombreux est désertée. La même minimisation des faits intervient dans la station d'Ilfracombe afin de limiter les dommages à l'activité touristique. Jusqu'à la fin de la saison estivale, le North Devon Journal continue d'expliquer que les rumeurs sont, dans bien des cas, des pures inventions. Il faut finalement attendre le mois d'octobre pour qu'un officier local annonce que, si rien n'est fait d'ici le mois de mai, ce sera la ruine pour de nombreuses personnes. Après l'envoi d'une pétition des autorités locales, un rapport, rédigé par Thomas Rammell, confirme l'état d'hygiène déplorable des stations balnéaires de Torquay, Ilfracombe et Exmouth (qui a échappé à la pandémie).

\section{Un secteur touristique distrait des enjeux de santé au $\mathrm{XX}$ siècle}

17 Au tournant du $x^{e}$ siècle, la maitrise des risques médicaux - au moins dans les pays occidentaux - continue de s'améliorer, la mort devenant davantage une affaire de "mauvaise chance statistique » et de destinée singulière que celle d'une catastrophe collective. Preuve de la grande confiance dans la maîtrise du risque épidémique, le médecin Charles Nicolle, dans son ouvrage Naissance, vie et mort des maladies infectieuses (1930), se sent obligé d'annoncer qu'il sera quand même difficile d'éradiquer les maladies (cité par Coquin et Chemardin, 2008). Le tourisme connaît alors un développement serein, de plus en plus distrait des crises sanitaires même si leurs effets, à l'échelle locale, peuvent être particulièrement dommageables. Avec l'essor des transports rapides et le "rapprochement» des destinations exotiques, le touriste redevient toutefois, dans les mentalités, un possible colporteur de maladies.

\section{Des territoires à la pointe sanitaire qui, définitivement, ne sont plus des espaces de refuge sanitaire}

$\mathrm{Au}$ début $\mathrm{du} \mathrm{xx}^{\mathrm{e}}$ siècle, le contrôle n'est plus policier, il est désormais médical, et l'autorité est devenue scientifique selon un principe nouveau: le virus n'a pas de frontières. La riposte sanitaire se double d'un pouvoir d'injonction, par exemple en France à travers la grande loi de santé publique du 15 février 1902. Il ne s'agit plus de faire rempart. C'est ainsi que, dans le cadre du processus d'hygiénisation des sociétés occidentales (de 1870 à la veille de la guerre de 1914), les États travaillent à améliorer l'assainissement dans les villes et villages, et les lieux touristiques n'échappent pas à ce volontarisme, inscrit notamment dans la loi de 1902 en France, d'autant qu'ils sont considérés comme incompatibles avec l'idée même d'insalubrité (Fijalkow, 2000 ; Frioux et al., 2011). Dès lors, l'hygiène devient l'un des premiers, sinon le premier, outils 
d'intervention de l'État dans l'aménagement des stations touristiques. En France, les enquêtes nationales de 1908 et 1912 permettent de déterminer la nécessité d'une intervention forte, qui se traduit notamment par l'adoption de la « cure taxe » en 1910 (ancêtre de la taxe de séjour, permettant de financer les équipements sanitaires et les embellissements urbains), sur les modèles allemand et austro-hongrois. Cette dernière devient la contrepartie de l'obligation d'aménagements en faveur de la salubrité et de l'embellissement pour (entre autres) les communes touristiques, imposée en 1919 par la loi Cornudet (qui sera complétée en 1924). La préparation législative de cette loi, en 1914, avait finalement été repoussée à cause du contexte de guerre (Vincent, 2007).

19 La station - qu'elle soit balnéaire, thermale ou de montagne - n'est définitivement plus un lieu «magique » qui justifie de s'y réfugier en cas d'épidémie ou de pandémie. À ce titre, la grippe espagnole confirme la tendance amorcée à la fin du siècle précédent : la mythologie sanitaire universelle des stations touristiques est définitivement révolue, à de très rares exceptions, notamment au Brésil (Faber do Nacimiento, 2020). Ainsi, les revues spécialisées, à l'image de La Revue du Touring-Club de France, n'évoquent aucune recrudescence d'un tourisme sanitaire lors de l'hiver 1918-1919. La grippe espagnole n'y est d'ailleurs pas évoquée, alors qu'elle fait l'actualité de la presse nationale. Au contraire, le Touring-Club de France insiste auprès des acteurs du tourisme de montagne pour qu'ils ne ratent pas l'opportunité qui se présente à eux de vanter les mérites des stations de ski françaises auprès des soldats britanniques et américains. Désormais, si les espaces touristiques demeurent des espaces de refuge, ce n'est plus pour des raisons sanitaires mais pour des raisons de sécurité et de confort, notamment lors des conflits armés (Evanno et Vincent, 2019).

\section{La pandémie, inoffensive pour le tourisme?}

Affranchies des réponses policières qui entravaient la liberté de circuler et donc de voyager, les mobilités touristiques ne semblent plus souffrir des épidémies et des pandémies au sortir de la Grande Guerre. Si le faible impact de la grippe espagnole sur le tourisme reste certes difficilement interprétable en raison du contexte international (fin de conflit, épidémie ayant frappé l'Europe lors de l'automne et de l'hiver 1918-1919), les quatre pandémies qui se succèdent en l'espace de vingt-cinq ans après la Seconde Guerre mondiale ( $c f$. tableau 1) semblent confirmer le peu d'influence des pandémies sur le tourisme international.

Tableau 1. Pandémies des années 1950 et 1960

\begin{tabular}{|l|l|l|l|}
\hline Dates & Nom donné à la pandémie & Surmortalité en France & Surmortalité dans le monde \\
\hline $1948-1949$ & Grippe italienne & 36000 & chiffre inconnu \\
\hline 1953 & Grippe de 1953 & 28000 & chiffre inconnu \\
\hline $1956-1958$ & Grippe asiatique & 10000 & entre 1000000 et 4000000 \\
\hline $1968-1969$ & Grippe de Hong-Kong & 25000 à 31000 & 1000000 \\
\hline
\end{tabular}

Sources: Trainar, 2020, p. 256 ; site internet de l'OMS 
21 pandémies sur les pratiques touristiques reste encore mal connue. Il ressort cependant quelques tendances. Ainsi, la gestion de crise, sous l'égide de l'Organisation mondiale de la Santé (OMS), s'évertue à rassurer la population, comme en France en 1953 où la pandémie est présentée comme bénigne malgré « une sérieuse manifestation de la grippe » (Anonyme, 1953). Des actions de prévention sont également entreprises durant ces épisodes pandémiques : en 1948, projet d'étendre les vacances scolaires de Noël en Italie pour limiter la contagion; en 1957, embauche des opératrices en formation dans les télécommunications en Suisse, don de sachets contenant des comprimés pour lutter contre les atteintes de la grippe pour les vendeuses de Genève (Anonyme, 1957); en 1969, promotion du masque par des starlettes en Belgique... Il s'agit d'abord de rassurer sur la bonne gestion de la crise, tout en suscitant une forte mobilisation internationale, coordonnée par l'OMS, avec les débuts des premiers vaccins antigrippaux efficaces, dès novembre 1968 (Sardon, 2020).

Lors de chacun de ces épisodes pandémiques, aucun message de sensibilisation spécifique n'est formulé dans les médias ${ }^{1}$ à l'attention des touristes. Considérée comme la première pandémie de l'ère moderne, celle des transports aériens rapides, la grippe de Hong Kong (1968-1969) ne sera présentée qu'a posteriori comme la première pandémie auquel est confronté le secteur touristique international (Sardon, 2020). En effet, au moment de la crise, la relation entre épidémie et tourisme est rarement évoquée, hormis quelques entrefilets à l'image de celui paru en janvier 1969 dans le Journal de Genève qui rapporte une information de l'OMS selon laquelle une poussée de grippe de Hong Kong était survenue à Davos au sein d'un groupe de touristes en provenance de Los Angeles (Anonyme, 1969). De la même manière, malgré 12 millions de malades et 31000 morts en France en décembre 1969 et janvier 1970 (bilan précis réalisé seulement en 2003 : voir Sardon, 2020), l'épidémie passe quasiment inaperçue dans la presse hexagonale. Il faut parfois se référer à des entrefilets et à des articles très locaux pour en trouver mention. Ainsi, début janvier 1970, une enquête locale menée par Ouest-France à Angers révèle " qu'une baisse sensible de départs vers la montagne " a été observée dans la ville, précisant que " des réservations ont dû être annulées au dernier moment en raison des grippes » (Anonyme, 1970).

Aucune source formelle ne nous permet, à ce jour, d'expliquer ces situations paradoxales. Dans les archives que nous avons consultées, aucun plan de gestion de crise par les acteurs du tourisme n'est évoqué. L'exemple des inventaires des Archives nationales (en France) parle de lui-même : aucune liasse comportant des documents d'avant 1993 n'est décrite avec la recherche "pandémie/grippe », la recherche "grippe asiatique " ne renvoie comme réponse pertinente que le fonds déposé du service du médecin-chef des PTT (liasse sur les grippes coté 19890032/10), tandis que la recherche " grippe de Hong Kong » reste sans résultats. Cette invisibilité des sources n'étonnera pas le chercheur qui s'intéresse au tourisme en temps de crise puisque les classements archivistiques ne prévoient pas de classements thématiques, ce qui rend la recherche plus longue, incertaine et - parfois - décourageante (Evanno et Vincent, 2019). Les informations sont donc disséminées dans une grande diversité d'archives produites par une multitude de services administratifs et d'acteurs privés et publics.

En conséquence, nous nous en tiendrons, à ce stade, à la formulation d'hypothèses. On peut en effet se demander si, dans le souci de préserver la respectabilité d'une destination, le niveau local ne s'empare pas de la gestion de la crise sanitaire autrement 
que contraint, puisque toutes les autorités (aussi bien économiques que politiques) sont confrontées au problème et qu'aucune n'a intérêt à communiquer sur le sujet. En effet, la prise de conscience d'une potentielle crise sanitaire, en lien avec une épidémie ou une pandémie, se fait dans des espaces très localisés et la crise est gérée, au moins dans un premier temps, avec des moyens et des objectifs locaux. Dès lors, la communication des niveaux national et international, arguant d'une confiance nécessaire par l'application de règles d'hygiène, ne trouve pas de relais locaux. Il ne faut en effet par perdre de vue que c'est aussi la démultiplication des lieux contaminés (dénommés «clusters» en 2020) dans une région (pour l'épidémie) ou dans le monde (pour une pandémie) qui transforme la crise sanitaire locale en crise générale (pouvant potentiellement corrompre l'ensemble de l'économie et avoir des conséquences sur la sûreté générale). Dans le cas d'une pandémie, où le monde entier est malade, pourquoi en faire localement plus que le voisin, au risque de faire fuir le touriste vers une autre destination tout autant concernée par la pandémie? Les médias et les acteurs nationaux et internationaux s'emparent de ces enjeux, qui émanaient jusqu'alors du seul prisme local; d'où l'importance, pour l'historien, d'adopter une vision multiscalaire, où prédomine l'échelle locale.

\section{Vers un état de crise sanitaire permanent ?}

25 À partir des années 1960, notamment en raison du «décollage touristique » (Cazes, 1989, p.63-66) de nombreux pays, le tourisme s'est retrouvé confronté à un risque permanent : celui des crises sanitaires endémiques. Ainsi, dans les années 1970, comme le met en évidence le film Les Bronzés qui se déroule dans un club de vacances en Côte d'Ivoire, les Européens sont de plus en plus nombreux à se laisser tenter par des destinations tropicales, en Amérique du Sud, en Afrique et en Asie. En 1979, le quotidien Ouest-France s'inquiète de ces "nouveaux risques du tourisme» à l'observation de la multiplication des cas de malaria et de fièvres jaunes en Europe, maladies principalement contractées à l'occasion de voyages en Afrique tropicale. Pour le journal, la situation est d'autant plus " préoccupante » que " rares sont les médecins ayant soigné ou vu ces maladies » (Anonyme, 1979, p. 6). En effet, la création, en 1963, d'Europ Assistance par Pierre Desnos et André Rosa (Obadia et Cha, 2020), organise désormais le rapatriement des malades dans leur pays d'origine sans attendre la fin de leur voyage. Dans ces conditions, les médecins se trouvent parfois dépourvus de solutions, comme ce fut le cas pour ces deux touristes, âgés de 24 ans et de 47 ans, décédés de la fièvre jaune à Paris après avoir séjourné dans un centre de vacances du Club Méditerranée au Sénégal (Anonyme, 1979). Cette situation perdure jusqu'à nos jours : le Bulletin épidémiologique hebdomadaire a ainsi publié en 2007 une étude sur les cas importés de choléra en France, constatant le nombre croissant de voyageurs français, pour partie âgés ou en bas âge, pour partie en contact étroit avec la population locale, alors que le choléra reste une maladie méconnue des médecins urgentistes ou généralistes (Tarantola, 2007).

Les structures internationales paraissent mieux armées. Les pandémies $d u x^{e}$ siècle ont eu comme conséquence la création de structures en charge de la santé à l'échelle mondiale. La grippe espagnole incite la Société des Nations (SDN), nouvellement créée (le 10 janvier 1920), à instaurer en son sein l'Organisation d'hygiène de la SDN, ancêtre de l'OMS (créée en 1948). Mais, jusqu'à la fin du xx siècle, l'OMS a des prérogatives limitées, qui s'étendent progressivement à mesure que se succèdent les pandémies. À 
partir de 1951, il existe certes un Règlement sanitaire international (RSI), juridiquement contraignant, mais, là-aussi, d'une portée limitée. Suite à la grippe de Hong-Kong, il requiert une communication des pays seulement dans les cas des maladies dites quarantenaires (impliquant une quarantaine) précisément désignées : la variole, le choléra, la fièvre jaune, le typhus, la peste et la fièvre récurrente (Coronado Martínez , 2016).

\section{Impact localisé et respectabilité des territoires}

Si les pandémies paraissent avoir paradoxalement peu d'impact sur le tourisme international au $\mathrm{xx}^{\mathrm{e}}$ siècle, les épidémies touchent en revanche durement les lieux touristiques. Après-guerre en effet, outre les pandémies, il y a eu de multiples épidémies, qui ont toutes donné lieu à des réactions des acteurs touristiques. Le réflexe local de minimisation d'une épidémie, entrevu au XIX ${ }^{e}$ siècle, se renforce alors considérablement à mesure que se popularise le secteur touristique, notamment en raison des retombées économiques qu'il génère et d'une internationalisation croissante des pratiques (Cuvelier, 1998, citant Rozenberg, 1990). Après-guerre, l'économie touristique est devenue vitale et indispensable pour de nombreuses destinations, en particulier dans les pays occidentaux.

L'épidémie, lorsque ses effets sont connus, oblige à réagir rapidement car toutes les régions du monde ne sont pas touchées. Cela constitue le meilleur moyen de lutter contre la rumeur, qui altère immédiatement l'imagerie touristique, et les conséquences néfastes qu'elle peut avoir sur l'économie. Les acteurs en sont pleinement conscients, comme le souligne le service des renseignements généraux du Morbihan au début de l'année 1955. Alors que la variole touche la Bretagne, un rapport indique, à propos des "étrangers du département ", que ces derniers :

paraissent encore plus méfiants [que les non-Vannetais] en raison surtout des rumeurs colportées dans le public selon lesquelles l'accès du département est contrôlé ou même interdit. Un Vannetais rapporte que, de passage en gare de Dax, il y a vu une affiche demandant aux voyageurs de ne pas aller dans le Morbihan: Il y avait danger de mort à cause de la variole. (Archives départementales du Morbihan, 1526 W 26, note d'information $n^{\circ} 66,27$ janvier 1955)

Pareille menace touche Zermatt au printemps 1963. La station touristique suisse fait les gros titres de la presse nationale et internationale en raison de l'épidémie de fièvre typhoïde qui vient entacher sa réputation de source de santé : trois personnes en décèdent et 450 sont hospitalisées. L'affaire est désastreuse pour l'image de la station (Kreis, 2014). L'historien Eberhard Wolff (2014) constate que l'épidémie de Zermatt se déroule dans une Suisse où l'image de propreté et de bonne santé appartient à la perception nationale du pays. La station tente sans tarder de rassurer les touristes. En effet, si ces derniers ne recherchent pas particulièrement un séjour thérapeutique de leur venue en montagne, ils ne souhaitent certainement pas y perdre la santé, voire la vie. Pour éviter une mise en cause et une éventuelle souillure indélébile, le rejet se fait souvent envers l'«autre». Ainsi, la station désigne immédiatement un saisonnier italien comme étant le "patient 0 », avant que l'état des structures d'eau potable ne soit mis en cause. Ce réflexe interroge la dualité du rapport que le pays entretient vis-àvis des étrangers : une attitude ouverte quand ceux-ci voyagent dans le pays en tant que touristes mais aussi, parallèlement, une défense instinctive à leur encontre quand, en tant que saisonniers, ils amènent avec eux des problèmes en plus de leur force de 
travail. Eberhard Wolff (ibid.) estime qu'« on » voulait alors surtout se tourner vers l'avenir. La communication s'est avérée réussie puisque, dès le début de l'été suivant, les pré-réservations pour la saison d'hiver étaient déjà meilleures que jamais.

La mise en concurrence exacerbée des destinations ne fait que renforcer la nécessité de préserver la respectabilité des territoires, et ce même si cela se fait au détriment des dispositions sanitaires. Dans les années 1970, le démographe Jacques Houdaille (1975) s'inquiète du manque d'informations fournies aux voyageurs au sujet des maladies tropicales. En ne cherchant pas à détecter efficacement les cas, les pays évitent, selon lui, de jeter le discrédit sur leur territoire: «il est évident que ceux qui tirent une grande partie de leurs ressources du tourisme n'ont pas intérêt à se montrer rigoureux à cet égard » (op. cit., p. 142). À ce titre, il explique que si la vaccination peut apparaître comme une solution, c'est au risque de stigmatiser un territoire et de faire fuir les potentiels voyageurs. Particulièrement médiatisé, le drame entourant la mort des deux touristes français provoque, en 1979, une prise de conscience dans la presse française, mais certains pays continuent de ne pas imposer de vaccination contre la fièvre jaune ou d'accorder des exceptions pour les courts séjours touristiques, comme au Sénégal, de façon à faire "tomber les freins au tourisme" (dans Ouest-France; Anonyme, 1979, p.6) ou « sous le prétexte que leur séjour serait inférieur à deux semaines » (dans Le Monde ; Lafaix, 1990, p. 21).

31 À la fin des années 1980, beaucoup d'agences de voyages, "soucieuses sans doute de ne pas effrayer les touristes potentiels » selon le journaliste Christian Lafaix (1990) qui consacre un dossier à la question dans les colonnes du Monde en 1990, continuent «d'oublier» de conseiller la vaccination à leurs clients. Il prend pour exemple les brochures touristiques qui comportent "des omissions ou des erreurs ", à l'image de l'édition 1989-1990 du guide professionnel des voyages et du tourisme ICOTOUR qui ne signale pas la recommandation du vaccin contre la fièvre jaune aux voyageurs désirant se rendre au Sénégal. Selon Christian Lafaix, la raison de ces omissions est simple : les pays concernés sont généralement peu soucieux de se livrer à des contrôles, pour les mêmes raisons que celles déjà évoquées par Jacques Houdaille quinze ans plus tôt.

\section{De l'aléa de la crise sanitaire au risque sanitaire touristique}

Depuis la fin du $\mathrm{xx}^{\mathrm{e}}$ siècle, l'existence des épidémies et des pandémies, et le risque qu'elles représentent dans le cas d'une fréquentation touristique, entraînent deux formes de réponses : une « nécessaire » information des voyageurs, d'une part, qui peut aussi être soupçonnée de patriotisme touristique sous-jacent alors que la clientèle, en Occident, est mieux éduquée; et une action permanente pour éviter les situations sanitaires incontrôlables. En effet, désormais, la globalisation du marché touristique transforme une catastrophe localisée dans une partie du monde en crise touchant l'ensemble de la planète, si bien que les crises contemporaines ne reconnaîtraient - ou ne respecteraient - plus les frontières nationales et ne se limiteraient plus à un secteur en particulier, comme la santé ou l'énergie par exemple (Maditinos et Vassiliadis, 2008). C'est pourquoi, en matière de tourisme, la gestion de crise se veut mondiale, alors qu'elle demeure, dans les faits, le ressort des acteurs locaux. 


\section{Nouvelle succession de pandémies à partir des années 1990 et prise de conscience}

À partir du milieu des années 1990, la succession des pandémies (cf. tableau 2) participe à la prise de conscience, par les États, du fait que les crises sanitaires exigent désormais une réponse de tous les secteurs et de tous les niveaux de gouvernement.

Tableau 2. Pandémies des années 2000 et 2010

\begin{tabular}{|l|l|l|l|}
\hline Dates & Nom donné à la pandémie & Surmortalité en France & Surmortalité dans le monde \\
\hline Depuis 1997 & Chikungunya & au moins 203 & chiffre inconnu \\
\hline 1997 & Grippe H5N1 de Hong-Kong & 0 & 282 \\
\hline 2003 & SRAS & 1 & environ 800 \\
\hline $2005-2006$ & Grippe aviaire & 0 & environ 100 \\
\hline 2009 & Grippe H1N1 ou mexicaine & 323 & 200000 \\
\hline $2014-2016$ & Ebola & 0 & plus de 20000 \\
\hline $2012-2015$ & MERS & 0 & 458 \\
\hline
\end{tabular}

Sources : Trainar, 2020, p. 256 ; site internet de l'OMS Dès après les attentats du 11 septembre 2001, dans la crainte de nouvelles vagues d'attentats (l'arme chimique est clairement identifiée comme menace principale par les États dans les conflits asymétriques: voir Lion, 2009), les organisations nationales et mondiales de santé sont encouragées à coopérer avec la mise en place de la Global Health Security Initiative (GHSI), à l'initiative des États-Unis. Ces coopérations se déclinent ensuite régionalement: le North America Plan for Avian and Pandemic Influenza (NAPAPI) est révisé pour mieux préparer les systèmes de santé (Coronado Martínez, 2016). Le RSI est revu en 2005 (dans le cadre d'un processus initié en 1995) afin de prendre en compte tout type d'épidémie et de mettre en œuvre une action coordonnée et proportionnée, en évitant de créer des entraves inutiles au trafic et au commerce internationaux. Plus que contre l'épidémie elle-même, la coopération internationale souhaite lutter contre la stupeur.

En 2006, à Berlin, lors de l'International Tourism Bourse, la menace de grippe aviaire est abordée par l'OMS dans une réunion d'information pour préparer les professionnels (Anonyme, 2006). Si la gestion de la crise du H5N1 a rassuré les autorités, l'apparition d'une pandémie grippale associée au virus H1N1, en 2009, inquiète davantage, notamment les acteurs du secteur touristique qui en subissent de plein fouet les conséquences. Au Mexique, les touristes états-uniens et canadiens annulent leur séjour et les agences de voyages (TUI Travels, Thomas Cook et Virgin Holidays) cessent de vendre leurs circuits organisés. Les managers deviennent alors la cheville ouvrière de l'information sanitaire, tant à destination de leur personnel que de leurs clients. Le flux 
de voyageurs aériens internationaux diminue de 20 à $25 \%$ au Mexique (CordovaVillalobos, 2010, cité par Coronado Martínez, 2016). Le secteur touristique y perd 10,5\% de ses revenus par rapport à l'année précédente. Ces chiffres sont néanmoins à analyser avec prudence, comme le rappellent, déjà à l'époque, de nombreux observateurs, car cette crise sanitaire est concomitante de la crise économique de 2008. Il n'en demeure pas moins qu'une prise de conscience a lieu quant à la vulnérabilité du tourisme face aux pandémies, désormais présentées comme des épisodes potentiellement récurrents (Anonyme, s.d.; Anonyme, 2009).

C'est dans cette optique que l'Organisation mondiale du tourisme (OMT/WTO) organise deux journées d'études les 26 et 27 août 2009, sous le titre « Travel and Tourism under Pandemic Conditions » (UNWTO Headquarters, 2009). Si les participants admettent que le risque pandémique demeure important, ils se félicitent de l'expérience acquise durant la crise sanitaire du H5N1 en 2006. Ces crises servent de base de réflexion pour formuler aux professionnels du tourisme de nombreuses préconisations en cas de nouvelle pandémie afin de responsabiliser le touriste, maintenir un équilibre entre des mesures préventives et l'incitation à continuer de voyager et établir des plans de continuité d'activité dans les entreprises touristiques. Les différents acteurs s'accordent très largement sur le fait de ne pas recommander de restrictions de voyage ni de fermeture de frontières.

Ce mode de gestion de crise, sous la forme de plans que les gouvernements sont enjoints de formuler, de suivre et de respecter, entraîne une autre manière de penser la crise et une autre appréhension de l'activité économique. Or les pandémies ont comme caractéristiques, dans le cas de nouveaux virus, d'être impossibles à anticiper (Bricaire, 2008).

\section{D'une approche locale au risque du jugement international}

De la théorie à la pratique, l'écart s'avère parfois trop grand. Dans les faits, face aux crises sanitaires, les États et les acteurs socio-économiques se retrouvent dans une position ambivalente. Confrontés à une coopération internationale qui milite pour une diffusion la plus large possible des informations, ils doivent en même temps gérer les situations épidémiques et économiques localement. L'article 43 du RSI de 2005 sur la libre circulation des personnes et des biens fait très tôt l'objet de contestations de la part des États (Hoffman, 2010). Le secteur du tourisme, dont s'emparent les organismes et les États, à différentes échelles, est alors tiraillé. En effet, communiquer sur une crise risque de jeter le discrédit sur un territoire.

En matière de tourisme, les approches pratiques des crises sanitaires peuvent donc apparaître contradictoires, avec des jeux d'acteurs qui évoluent et s'opposent. Le cas mauricien de gestion de l'épidémie de Chikungunya en 2006-2007 peut servir d'exemple. En mars 2006, les autorités de Maurice s'inquiètent des conséquences de la maladie du Chikungunya sur le tourisme et invitent l'OMS à enquêter. Cette dernière conclut qu'il ne s'agit pas d'un problème majeur de santé publique. L'île Maurice cale sa communication touristique sur ce verdict, qui rassure les acteurs touristiques. Un an plus tard, la diffusion du virus en Italie durant l'été 2007 conduit à un changement de perception de la part de l'OMS, la maladie étant désormais jugée sévère. Mais l'île Maurice ne modifie pas fondamentalement sa position de communication. Si les chercheurs estiment avoir partagé les quelques informations qu'ils avaient à leur 
disposition durant cette crise sanitaire, l'épidémiologiste Antoine Flahault (2009), dont il faut préciser qu'il est juge (avec l'article qu'il écrit) et partie (en tant que médecin impliqué dans la gestion de la crise sanitaire), note que «la tentation des autorités de l'île Maurice de minimiser l'épidémie pour ne pas trop en subir les conséquences au niveau du tourisme s'est traduite possiblement par un nombre plus élevé de personnes atteintes par la maladie».

Les acteurs du tourisme redoutent d'autant plus le discrédit que, lorsqu'un territoire est touché par une épidémie, ce sont désormais les régions proches et l'ensemble du pays concerné, voire le continent, qui en subissent également les conséquences (Carter, 1998). En avril 2003, alors que Toronto est touché par plusieurs cas de SRAS (16 morts à cette date), l'OMS décide de placer la ville canadienne sur «la liste noire des pays à éviter ", au même titre que Hong Kong et Pékin. C'est bien plus que l'image touristique de la ville qui est atteinte. Immédiatement, les autorités locales et nationales condamnent le communiqué de l'OMS, craignant qu'il ne fasse une mauvaise promotion touristique de l'ensemble du pays. Quelques jours avant la déclaration de l'OMS, Pierre Bellerose, porte-parole de Tourisme Montréal, s'inquiétait déjà de la mauvaise publicité faite à son pays depuis la découverte de plusieurs cas: «nous n'avons pas encore de chiffres, mais c'est préoccupant. Les touristes d'Europe ne font pas toujours la distinction entre Montréal et Toronto. Tout ce qu'ils savent, c'est que nous sommes au Canada » (Anonyme, 2003).

41 Dix ans plus tard, en 2014, le virus Ebola affecte de la même manière le tourisme du continent africain alors même que l'épidémie ne touche essentiellement que trois pays (Guinée, Sierra Leone, Liberia). À titre d'exemple, au Kenya, la fréquentation touristique baisse de 7,7 \% au cours de l'année (Ildiko et Zsuzsanna, 2020). Exaspéré par cette situation, le directeur de l'office du tourisme de Namibie rappelle aux touristes européens qu'ils sont plus proches de l'épicentre de l'épidémie d'Ebola que la Namibie elle-même (Anonyme, 2015; Anonyme, 2016). À l'échelle mondiale, selon un commentateur de l'époque, «le principal impact du virus Ebola sur les voyages a été observé à la bourse. Les actions des entreprises du secteur et des compagnies aériennes ont perdu quelques points sur toutes les places financières début octobre. Mais les analystes boursiers ne s'attendent pas à ce qu'Ebola provoque les mêmes effets que l'épidémie de SRAS en 2003 » (Jarre, 2014). De la même manière, dans un article au titre pourtant équivoque («Ebola menace-t-il l'industrie touristique?»), la revue L'Écho touristique se veut rassurante (Anonyme, 2014).

\section{À la recherche des bulles de safe tourism ?}

Afin de rassurer les touristes, les acteurs du secteur mettent en place des bulles propices au safe tourism. Ces dernières ne sont pas spécifiques aux crises sanitaires puisqu'elles sont également pensées pour contrer les risques naturels ou les menaces terroristes. Ainsi, la crise sanitaire de 2020, à peine commencée, était déjà considérée comme une rupture par certains auteurs, convaincus que cette situation devrait faire changer l'attitude des touristes qui, désormais conscients du risque, seraient disposés à privilégier des lieux de vacances qui leurs assureraient une sécurité sanitaire et médicale (Ildiko et Zsuzsanna, 2020). Alors que les bulles touristiques étaient, dans les années 1990, une valorisation spécifique entre un imaginaire et un espace touristique 
(Remy, 1994), il s'agirait, depuis les années 2000, de faire évoluer le visiteur dans une bulle pacifique ou/et pacifiée (Dehoorne, 2013).

La recherche de la confiance est telle qu'elle se traduit par des promesses de compensation en cas de problème. Ce type de proposition était déjà survenu lors de la crise sanitaire à Zermatt en 1963, durant laquelle les professionnels promettaient, en cas de maladie contractée sur site, trois semaines de vacances dans une station suisse de leur choix, les frais de transport et d'hôtel étant à la charge de l'office suisse du tourisme, de la Société suisse des hôteliers, du Syndicat d'initiative et de la Société des hôteliers de Zermatt. Des mesures avaient également été prises en faveur du personnel tombé malade (Anonyme, 1963). Mais cette pratique demeure exceptionnelle au $\mathrm{xx}^{\mathrm{e}}$ siècle et la réalité de ce type de dédommagements n'est pas connue. Elle devient en revanche récurrente à partir de la fin des années 2000. Ainsi, en 2009, une dépêche de l'AFP précise que «le Mexique est frappé de plein fouet par les effets du virus H1N1: des hôtels de la célèbre station balnéaire de Cancún, désertés par leurs clients, sont allés jusqu'à offrir des vacances gratuites pendant trois ans aux touristes qui y seraient contaminés par la grippe porcine » (Hagemann, 2009). Onze ans plus tard, en mai 2020, alors que l'Europe sort à peine d'un premier confinement de sa population, des pays comme Chypre ou l'Ouzbékistan s'engagent à prendre en charge le coût des vacances de tous les touristes qui contracteraient le coronavirus sur leur sol (à hauteur de 3000 \$ pour l'Ouzbékistan).

Cette propension au dédommagement est nouvelle dans sa systématicité au début du $\mathrm{xxI}^{\mathrm{e}}$ siècle. Elle peut être pensée comme une conséquence de l'assurantialisation de la société (en Occident du moins), qui débute au XvIII siècle mais qui connaît depuis les années 1990 une forte progression, dans le cadre d'un vaste processus de dérégulation et d'homogénéisation des institutions, avec des politiques d'assurance au sens large. À l'origine pensée comme un instrument de protection, l'assurance est désormais perçue comme un bien premier, condition d'accès à une série de biens dont on ne conçoit plus d'être privé : c'est un principe d'organisation sociale (Ewald, 2011). Le tourisme entre dans la catégorie de ces biens premiers, pour une partie de la population occidentale tout au moins (Vincent, 2019).

Des stratégies marketing se mettent donc en place pour promouvoir la sécurité sanitaire d'une destination. En 2020, Monaco lance son label « Monaco safe ", destiné à faire savoir que la destination a pris des mesures pour protéger les intérêts de tous, initiative relayée par les professionnels du secteur touristique. Le surcoût du protocole sanitaire durant le printemps 2020 est évalué entre 10 et 15000 euros pour le Fairmont Monte-Carlo, somme que son directeur Xavier Rugeroni n'estime "pas énorme " (Anonyme, 2020, p. 40). Quant au " portail officiel du tourisme en Espagne", il a tout simplement pris en 2020 le nom de "Travel Safe » (spécifié clairement dans l'adresse du site: travelsafe.spain.info) afin de montrer que, pour tout type de pratique touristique, le pays a envisagé une solution sanitaire qui permet d'assurer aux touristes des vacances sereines. Mais cette réponse aux crises sanitaires n'appartient qu'aux territoires et aux acteurs qui ont les moyens financiers et humains d'investir.

Faut-il voir dans ces évolutions des mobilités touristiques, qui se réaliseraient de plus en plus au profit de territoires sécurisés depuis les attentats des années 2001-2002 (Dehoorne, 2013), une marginalisation progressive de la majorité des destinations émergentes tant ces politiques impliquent des apports financiers importants ou une situation sécurisée à l'origine? 


\section{Conclusion}

Avec la crise sanitaire qui s'est ouverte en 2020, et qui n'est pas achevée au moment où nous écrivons ces lignes, s'ouvre un nouveau champ d'étude pour les chercheurs en tourisme qui, contrairement aux pandémies passées, est bien documenté. La Covid-19 a bien entendu entraîné une crise singulière pour le secteur touristique du fait, notamment, de la fermeture des frontières, de la remise en place de quarantaines et de la crainte de ne pas pouvoir être bien soigné dans le pays hôte. Gérée selon des préconisations d'abord locales (les clusters, les confinements locaux), avec des enjeux en matière de réputation (le virus « chinois » du président états-unien Donald Trump, le «virus de Courchevel » à cause de la contamination des élites ukrainiennes durant leur séjour...), la crise est devenue la justification de nouvelles interventions, notamment de la part des États, à l'encontre des réglementations internationales (comme le Règlement sanitaire international de 2005, en particulier au sujet de l'article insistant sur l'importance de ne pas restreindre la circulation et ne pas fermer les frontières, marginalisant ainsi - temporairement? - l'action jusqu'alors croissante de l'OMT). Mais la crise de 2020 reste, malgré tout, semblable à celles du passé sur de nombreux points: importance de ne pas entacher la respectabilité d'un territoire touristique; caisse de résonance de tendances déjà amorcées quelques années auparavant (Evanno et Vincent, 2020)... Il importe donc de poursuivre les efforts visant à mieux comprendre, de façon holistique, l'évolution historique du tourisme dans ce contexte complexe des crises sanitaires.

Indéniablement, comprendre la crise actuelle ne peut faire l'économie d'une étude sur le temps long permettant de mieux mesurer le rôle incontournable des acteurs locaux lors des crises épidémiques ou pandémiques. Malheureusement, la faible audience de leur action, car limitée géographiquement, ainsi que l'éparpillement des sources disponibles expliquent, en grande partie, pourquoi le lien entre tourisme et épidémies demeure un angle mort historiographique. Dès lors, il est parfaitement compréhensible que la mémoire du risque sanitaire soit encore très largement absente du secteur du tourisme. En cela, Gokhan Karabulut et ses co-auteurs (Karabulut et al., 2020) ont raison de penser que les crises sanitaires auraient des effets qui ne seraient pas durables, car aucune mémoire n'est là pour les rappeler. Il n'empêche, les crises du passé (sanitaires ou non) démontrent qu'elles génèrent, sur le temps long, de profondes mutations sociales et économiques dans le secteur touristique. En analysant les permanences qui émergent ou qui sont malmenées au moment des crises, cette étude permet de dépasser les limites imposées par une situation "où tout bouge en même temps", qui rend difficile la compréhension des origines d'une mutation.

Si la crise sanitaire mérite d'être étudiée en tant que telle, l'étude du lien entre tourisme et épidémies ne doit pas omettre que les conséquences des pandémies se mêlent parfois aux conséquences d'autres crises, qui peuvent survenir auparavant ou en même temps, à l'image de la grippe espagnole qui survient à la fin de la Première Guerre mondiale ou du virus H1N1 dont les bilans sont faussés du fait de la crise économique de 2008 concomitante. Déjà à l'époque, Geoffrey Lipman, sous-secrétaire général de l'OMT, mettait en garde sur le fait qu'il serait difficile de savoir quel a été l'impact réel de la "grippe porcine » sur les voyages : «qui pourra dire que tel ou tel touriste a renoncé à son voyage parce qu'il avait peur de la grippe ou parce qu'il ne 
pouvait pas payer les traites de sa maison " (cité par Hagemann, 2009). L'étude des relations entre tourisme et épidémies/pandémies demeure donc un champ historique complexe qui, du fait de l'épidémie de Covid-19, trouve une nouvelle audience et ne manquera pas, espérons-le, de susciter l'intérêt des chercheurs.

\section{BIBLIOGRAPHIE}

ANONYME, « The Impact of Epidemics and Pandemics Essay », UK Essays, s.d. [http://

www.ukessays.com/essays/tourism/the-impact-of-epidemics-and-pandemics-tourism-

essay.php].

ANONYME, « Faits divers », La Concorde du Morbihan, 26 août 1849.

ANONYME, L'Illustration, des 19 juillet et 26 juillet 1884 .

ANONYME, « D'après l'OMS, l'épidémie de grippe qui sévit actuellement en Europe est bénigne », La Sentinelle, 30 janvier 1953.

ANONYME, « La grippe étend ses ravages », Journal de Genève, 16 octobre 1957.

ANONYME, «L'épidémie de typhoïde. Conférence de presse à Berne », Journal de Sierre et du Valais central, 17 mai 1963.

ANONYME, « Grippe de Hong-Kong à Davos », Journal de Genève, 25 janvier 1969.

ANONYME, « Deux morts de la fièvre jaune à Paris », Ouest-France, 9 novembre 1979, p. 6.

ANONYME, « Les Angevins et les vacances d'hiver », Ouest-France (éd. Anjou), 2 janvier 1970, non paginé.

ANONYME, « Toronto, ville à éviter à tout prix, selon l'OMS », Le Devoir, 24 avril 2003, p. A1.

ANONYME, « Organisation mondiale du tourisme - À l'ITB 2006, l'OMT tiendra une réunion

d'information sur la grippe aviaire », News Press, 6 mars 2006, non paginé.

ANONYME, «Le virus H1N1, fléau du tourisme international », La Presse, 7 octobre 2009 [https:// www.lapresse.ca/voyage/nouvelles/200910/07/01-909314-le-virus-h1n1-fleau-du-tourismeinternational.php].

ANONYME, «Épidémies : ce que nous enseigne le passé ", L'Écho touristique, n 3126, 24 octobre 2014, p. 8-9.

ANONYME, « Les ravages d'Ebola sur le tourisme africain », CNews, 7 mars 2015 [https:// www.cnews.fr/monde/2015-03-07/les-ravages-debola-sur-le-tourisme-africain-700834]. ANONYME, « Pourtant épargnés par Ebola, Sénégal et Gambie ont perdu leurs touristes », RFI.fr, 27 décembre 2016 [http://www.rfi.fr/fr/afrique/20161227-ebola-senegal-gambie-tourismeeconomie-voyage-risque].

ANONYME, "Covid-19. Les hôteliers de Monaco veulent "voir la lumière au bout du tunnel" ", Monaco Hebdo, $\mathrm{n}^{\circ}$ 1157, 18 juin 2020, p. 36-40. 
ARCHIVES DE NICE, Épidémies et maladies contagieuses à Nice du Moyen Âge à nos jours, Nice, Archives municipales, 2020 [https://archives.nicecotedazur.org/dossier_pedagogique/epidemies-etmaladies-contagieuses-a-nice/].

Andrée BACHEMONT, «Cannes et les Anglais (1835-1930) », Recherches régionales. Alpes-Maritimes et contrées limitrophes, $\mathrm{n}^{\circ}$ 197, 2011 [https://www.departement06.fr/documents/Import/decouvrirles-am/recherchesregionales197-08.pdf].

Elizabeth BAXTER et David BOWEN, « Anatomy of Tourism Crisis: Explaining the Effects on Tourism of the UK Foot and Mouth Disease Epidemics of 1967-1968 and 2001 with Special Reference to Media ", International Journal of Tourism Research, p. 263-273, 2004 [https://doi.org/10.1002/jtr. 487].

Catherine BERTHO-LAVENIR, La roue et le stylo. Comment nous sommes devenus touristes, Odile Jacob, 1999.

Dominique BON, «Cholera Epidemics, Local Politics and Nationalism in the Province of Nice during the First Half of the Nineteenth Century ", in John Chircop et Francisco Javier Martinez, Mediterranean Quarantines, 1750-1914: Space, Identity and Power, Manchester University Press, 2018, p. 47-65.

Marc BOYER, L'invention de la Côte d'Azur. L'hiver dans le Midi, Éditions de l'Aube, 2002.

François BRICAIRE, « Anticiper la “pandémie grippale” ", Sécurité globale, n 3, p. 47-55, 2008

[https://doi.org/10.3917/secug.003.0047].

Simon CARTER, « Tourists and Traveler's Social Construction of Africa and Asia as Risky

Locations », Tourism Management, n 19, 1998, p. 349-358.

Georges CAZES, Les nouvelles colonies de vacances. Le tourisme international à la conquête du TiersMonde, L'Harmattan, 1989.

Jose-Angel CORDOVA-VILLALOBOS, «Introducción: la epidemia de influenza A (H1N1) en México », dans Jose-Angel Cordova-Villalobos, Jose-Luis Valdespino Gómez et Samuel Ponce de León Rosales (dir.), La epidemia de influenza 1/H1N1 en México, Editorial Médical Panamericana, 2010.

Yves COQUIN et Jacques CHEMARDIN, «Les maladies émergentes : illusion ou réalité ? ", Annales des Mines - Responsabilité et environnement, $n^{\circ}$ 51, 2008, p. 15-20.

María Esther CORONADO MARTÍNEZ, « The Mexican Experience of the NAPAPI Revision Process », Contexto Internacional, vol. $38, \mathrm{n}^{\circ} 1,2016$, p. 203-239.

Daniel CUVELIER, Anciennes et nouvelles formes de tourisme : une approche socio-économique, L'Harmattan, 1998.

Helen M. DAVIES, Emile and Isaac Pereire. Bankers, Socialists and Sephardic Jews in Nineteenth-century France, Manchester University Press, 2015.

Olivier DEHOORNE, « Une histoire du tourisme international : de la déambulation exotique à la bulle sécurisée ", Revue internationale et stratégique $\mathrm{n}^{\circ}$ 90, 2013, p. 77-85.

Jean DELUMEAU et Yves LEQUIN (dir.), Les malheurs du temps. Histoire des fléaux et des calamités en France, Larousse, 2020.

Alex EMPREZA, Petrópolis Cidade do Brasil : a Rainha das Serras, Tipografia da Escola Gratuita são José, 1910.

Yves-Marie EVANNO et Johan VINCENT, « Loin des tranchées, la plage. Réflexions sur le tourisme durant la grande Guerre à travers l'exemple du Morbihan », dans Yves-Marie Evanno et Yann 
Lagadec (dir.), Les Morbihanais à l'épreuve de la Grande Guerre (1914-1920), Département du Morbihan/Université Tous Âges, 2017, p. 177-202.

Yves-Marie EVANNo et Johan VINCENT (dir.), Tourisme et Grande Guerre. Voyage(s) sur un front historique méconnu, 1914-2019, Éditions Codex, 2019.

Yves-Marie EVANNo et Johan vinCENT, « Espoirs et désillusions des sorties de crise. De la nécessité d'être patient », Revue Espaces, n³ 355, 2020, p. 104-108.

François EWALD, « L'assurantialisation de la société française ", Les tribunes de la santé, $\mathrm{n}^{\circ} 31,2011$ p. 23-29.

Alan FABER DO NACIMIENTO, " The Tourist Topicality of the Case of the Spanish Flu in the City of Rio de Janeiro (Sep. 1918 - Mar. 1919) », Revista Brasileira de Pesquisa em Turismo, vol. 14, n³, p. $176-188,2020$

Yankel FIJALKow, « La notion d'insalubrité. Un processus de rationalisation, 1850-1902 », Revue d'histoire du XIX ${ }^{e}$ siècle, $\mathrm{n}^{\circ}$ 20/21, p. 135-153, 2000 [https://doi.org/10.4000/rh19.213].

Antoine FLAHAULT, « Gestion d'une crise sanitaire : l'exemple du chikungunya », Les tribunes de la santé, $\mathrm{n}^{\circ} 22$, p. 53-66, 2009 [https://doi.org/10.3917/seve.022.0053].

Lydia FLEM, « Bouche bavarde et oreille curieuse », Le Genre humain, n 5, p. 11-18, 1982.

Stéphane FRIOUX, Patrick FOURNIER et Sophie CHAUVEAU, Histoire et santé en Europe : de la fin du XVIII ${ }^{e}$ siècle aux années 1920, Éditions Sedes, 2011.

Anne-Marie GRANET-ABISSET, «L'autre "territoire du vide”. Des espaces répulsifs aux territoires préservés. L'exemple de la montagne alpine », dans Nathalie Richard, Renaud Morieux, Alain Cabantous, Jean-Luc Chappey et François Walter (dir.), Mer et montagne dans la culture européenne (XVI ${ }^{e}-\mathrm{XIX}{ }^{e}$ siècle), Presses universitaires de Rennes, 2011, p. 57-72.

Brigitte HAGEMANN, «L'industrie du voyage prise en étau entre la crise et le virus H1N1 », AFP Infos Mondiales, 15 mai 2009.

C. Michael HALL, «Crisis Events in Tourism: Subjects of Crisis in Tourism », Current Issues in Tourism, p. 401-417, 2010.

Philippe HANUS, Le choléra à nos frontières. Petite chronique de l'épidémie de 1884-1885 entre la France et l'Italie, Dossier de l'Ethnopôle du Cpa, avril 2020.

Steven J. HOFFMAN, « The Evolution, Etiology and Eventualities of the Global Health Security Regime ", Health Policy and Planning, vol. 25, n 6, p. 510-522, 2010.

Jacques HoudAILLE, « Le tourisme international et la maladie », Population, vol. 30, n 1, p. 140-142, 1975.

Norman HOWARD-JONES, Les bases scientifiques des Conférences sanitaires internationales, 1851-1938, OMS, 1975

Ernszt ILDIKo et Marton ZSUZSANNA, « Suspended and Closed. Effects of Epidemics on Tourism », dans Igor Klopotan, Katalin Czako et Victor Beker (dir.), Economic and Social Development, $53^{\text {rd }}$ International Scientific Conference on Economic and Social Development, édition en ligne, 2020, p. 61-69 [https://www.esd-conference.com/upload/book_of_proceedings/ Book_of_Proceedings_esdSvetiMartin2020_Online.pdf].

Stéphane JARRE, « Le tourisme à l'épreuve de l'actualité », Bus \& Car - Tourisme de groupe, $\mathrm{n}^{\circ} 39$, p. 10, 2014. 
Gokhan KARABULUT, Mehmet HUSEYIN BILGIN, Ender DEMIR, et Asli CANSIN DOKER, « How Pandemics Affect Tourism: International Evidence », Annals of Tourism Research vol. 84, 2020 [https://doi.org/ 10.1016/j.annals.2020.102991].

Georg KREIS (dir.), Die Geschichte der Schweiz, Schwabe, 2014.

Pierre LABORDE, Histoire du tourisme sur la Côte basque, Atlantica, 2001.

Christian LAFAIX, « Voyageurs sans vaccin », Le Monde, 28 novembre 1990.

Olivier LION, « Des armes maudites pour les sales guerres ? L'emploi des armes chimiques dans les conflits asymétriques », Stratégique n93-94-95-96, p. 491-531, 2009 [https://doi.org/10.3917/ strat.093.0491].

Zissis MADITINOS et Chrisyos VASSILIADIS, « Crises and Disasters in Tourism Industry: Happen Locally, Affect Globally », Management of International Business, p. 67-76, 2008.

Juan Carlos MONTERRUBIO, « Short-term Economic Impacts of Influenza A (H1N1) and Government Reaction on the Mexican Tourism Industry: An Analysis of the Media ", International Journal of Tourism Policy, 2010 [https://doi.org/10.1504/IJTP.2010.031599].

Pascal NOUVEL (dir.), Repenser le vitalisme. Histoire et philosophie du vitalisme, Presses universitaires de France, 2011.

Marina NOVELli, Liv Gussing BURGESS, Adam Jones et Brent W. RITCHIE, « No Ebola... Still Doomed. The Ebola-induced Tourism Crisis », Annals of Tourism Research, vol. 70, 2018 [https:// www.sciencedirect.com/science/article/pii/S0160738318300306].

Edouard OBADIA et Olivier CHA (dir.), Le rapatriement sanitaire par transport aérien médicalisé, à usage civil et militaire. Pathologies, techniques et réglementation, Elsevier Masson SA ; complété par les mêmes auteurs, de « Transports extra-hospitaliers : sociétés d'assistance », 2020 [www.tars.saintantoine.free.fr/Information.htm].

Roy PORTER, « Les Anglais et les loisirs », dans Alain Corbin (dir.), L'avènement des loisirs (1850-1960), Aubier, 1995, p. 21-54.

Jean REMY, « L'implication paradoxale dans l'expérience touristique », Recherches sociologiques, $\mathrm{n}^{\circ} 2$, p. 61-78, 1994.

Danielle ROZENBERG, Tourisme et utopie aux Baléares. Ibiza, une île pour une autre vie, L'Harmattan, 1990.

Jean-Paul SARDON, « De la longue histoire des épidémies au Covid-19 », Population \& Avenir, $\mathrm{n}^{\circ} 26$, p. 1-18, 2020.

STENDHAL, Mémoires d'un touriste, Le Divan, 1929 [1838].

Didier TABUREAU, «Les interdictions de santé publique », Les tribunes de la santé, $\mathrm{n}^{\circ}$ 17, p. 21-38, 2007.

Arnaud TARANTOLA, « Le choléra importé en France métropolitaine de 1973 à 2005 », Bulletin épidémiologique hebdomadaire, $\mathrm{n}^{\circ}$ 34, 2007 [http://beh.santepubliquefrance.fr/beh/2007/34/ beh_34_2007.pdf].

Sharon TEITLER-REGEV, Shosh SHAHRABANI et Oksana GOZIKER, « The Effect of Economic Crises, Epidemics and Terrorism on Tourism », International Journal of Business Tourism and Applied Sciences, vol 1, n 1, p. 84-89, 2013. 
Bernard TOULIER, « Architecture des villes d'eaux. Stations thermales et stations balnéaires », dans Villes d'eaux des Pyrénées occidentales. Patrimoine et devenir, Publications de l'Institut d'études du Massif Central, 1996, p. 23-36.

Philippe TRAINAR, «Les conséquences économiques du Covid-19 », Commentaire, n 170, p. 255-264, 2020.

John TRAVIS, The Rise of the Devon Seaside Resorts, 1750-1900, University of Exerter, 1993.

UNWTO Headquarters, Travel and Tourism under Pandemic Conditions, UNWTO, 2009.

Johan VINCENT, L'intrusion balnéaire. Les populations balnéaires bretonnes et vendéennes face au tourisme, Presses universitaires de Rennes, 2007.

Johan VINCENT, Here comes the Sun. Tourisme et mouvement des Gilets jaunes, Viens ! Éd. via LuluPress, 2019.

Marie-France VOUILLOZ-BURNIER, 1963, épidémie à Zermatt : la fièvre typhoïde à Zermatt, Éd.

Monographic, 2010.

Eberlhard WoLfF, « L'histoire de la médecine, une histoire suisse », Bulletin des médecins suisses, p. 1354, 2014.

\section{NOTES}

1. Presse et télévision, comme ce reportage diffusé par la télévision suisse du 15 novembre 1968 : https://www.rts.ch/archives/tv/divers/actualite-au-feminin/3445943-la-grippe-de-1968.html

\section{RÉSUMÉS}

Voyager à travers le monde n'est pas une activité sans risques. Si l'influence des aléas naturels, des conflits militaires ou encore des tensions sociales sur le tourisme international est relativement bien connue, il n'en va pas de même pour les risques sanitaires. La Covid-19, qualifiée de "sans précédent" pour le secteur touristique, a mis en évidence cette lacune historiographique, que cet article tente de commencer à combler par une approche sur le temps long, au-delà des seuls épisodes du $\mathrm{XXI}^{\mathrm{e}}$ siècle (SRAS, grippe aviaire, grippe porcine, MERS...). Alors que la maladie est perçue comme la conséquence d'un mauvais environnement jusqu'au $\mathrm{XIX}^{\mathrm{e}}$ siècle, plusieurs pandémies de choléra prouvent que même les lieux que l'on pensait alors préservés, permettant un tourisme de santé, peuvent être touchés. La gestion des pandémies modifie alors les intentions touristiques et favorise la prise de conscience que la respectabilité des territoires est devenue indispensable. Au tournant $\mathrm{du} \mathrm{xx}^{\mathrm{e}}$ siècle, la maîtrise des risques médicaux - au moins dans les pays occidentaux - continue de s'améliorer, la mort devenant davantage une affaire de «mauvaise chance statistique». Toutefois, avec l'essor des transports rapides et le "rapprochement" des destinations exotiques, le touriste redevient, dans les mentalités, un possible colporteur de maladies. En matière de tourisme, la gestion de crise se veut dès lors mondiale alors qu'elle demeure, dans les faits, le ressort des acteurs locaux. 
Traveling around the world is not a risk-free activity. While the influence of natural hazards, military conflicts, and social tensions on international tourism is relatively well known, health risks on tourism are still not properly understood. The Covid-19 pandemic, described as "unprecedented" for the tourism sector, has highlighted this historiographic gap that we wish to begin to fill with a long-term approach, beyond the $21^{\text {st }}$ century disease episodes (SARS, avian flu, swine flu, MERS...). While disease was perceived as the consequence of a bad environment until the $19^{\text {th }}$ century, several cholera pandemics prove that even places that were thought to be preserved, allowing for health tourism, can be affected. The management of pandemics raises awareness about the respectability of territories. In the early $20^{\text {th }}$ century, the control of medical risks - at least in Western countries - continued to improve, with death becoming more a matter of "statistical bad luck". However, with the rise of rapid transportation and the close contact with exotic destinations, the tourist once again became a possible carrier of the disease. In the field of tourism, crisis management is therefore intended to be global, although in reality it remains the responsibility of local actors.

\section{INDEX}

Keywords : pandemic, epidemic, health crisis, respectability, local actors, tourism history, Covid-19

Mots-clés : pandémie, épidémie, crise sanitaire, respectabilité, acteurs locaux, histoire du tourisme, Covid-19

\section{AUTEURS}

\section{YVES-MARIE EVANNO}

Enseignant dans le secondaire

Chargé de cours à l'Université catholique de l'Ouest Bretagne-Sud

Ymevanno[at]gmail.com

\section{JOHAN VINCENT}

Chercheur contractuel à l'Université d'Angers (UFR ESTHUA)

Chercheur associé à TEMOS (UMR 9016) et ESO (UMR 6590)

johan.vincent[at]univ-angers.fr 